\title{
Electronic transport through single-wall nicked carbon nanotubes
}

\author{
Wei Ren ${ }^{1}$ and Jian Wang ${ }^{1,2, *}$ \\ ${ }^{1}$ Department of Physics, The University of Hong Kong, Pokfulam Road, Hong Kong, China \\ ${ }^{2}$ Key Laboratory of Materials Physics, Institute of Solid State Physics, Chinese Academy of Sciences, Hefei, Anhui, China \\ (Received 10 April 2003; revised manuscript received 17 September 2003; published 30 January 2004)
}

\begin{abstract}
We investigate the electronic transport properties of single wall nicked carbon nanotube (SWNT) using a tight-binding model. In particular, the conductance, emittance, and local density of states of the nicked SWNT are calculated. The resonance structures at all energy levels depend, as expected, on the specific configuration of the system. Two transmissive eigenchannels through the system are separated and examined in detail. Several nicking strengths are considered and quantum dot behavior has been found only for heavily nicked carbon nanotubes. Furthermore, the interesting properties of the emittance of the nicked SWNT is discussed.

DOI: 10.1103/PhysRevB.69.033306

PACS number(s): 71.20.Tx, 73.63.Fg
\end{abstract}

Electronic transport through a single-wall carbon nanotube (SWNT) has received significant experimental and theoretical attentions ${ }^{1,2}$ since the discovery by Iijima. ${ }^{1}$ So far, most studies on SWNT related systems focus on dc transport. For instance, studies such as field effect transistors, ${ }^{3}$ SWNT ring, ${ }^{4}$ molecule-nanotube hybrids, ${ }^{5}$ and quantum $\operatorname{dots}^{6}$ have been demonstrated in the nanoresearch literatures. In a recent paper, Park et al. ${ }^{7}$ investigated the possibility to modify the electronic properties of nanotube devices with an atomic force microscope. By applying voltage pulses, the SWNTbased quantum device can be created along a tube. Electronic transport through this ultrasmall nanotube quantum device has been demonstrated in the experiment, the conductance as a function of the gate voltage showed a spiky behavior. Another important problem of current interest is the ac transport since any device operation will be under ac signals. When the system is under ac bias, two basic requirements have to be satisfied, i.e., the current conserving and gauge invariance. ${ }^{8}$ In the presence of ac field, the conduction current will not be conserved due the charge accumulation in the central scattering region. As a result, the induced displacement current has to be considered. ${ }^{9}$ Furthermore, the gauge invariance which means that the transport properties depend only on the voltage difference, will not satisfy for the ac transport properties. As pointed out by Buttiker ${ }^{10}$ that the physical origin behind these two requirements is the electron-electron interaction. When the frequency of external bias is small, the dynamical response of the system is characterized by the emittance ${ }^{11}$ which has been studied extensively for the mesoscopic system. ${ }^{12}$

Inspired by the experimental work of Park et al., we model the quantum system by simply extracting a few carbon atoms from the SWNT. We then study the electron transport in various configurations of nicked SWNT with nicks of different depth. Specifically, we have calculated the conductance, emittance, and the local density of states of the nicked SWNT. Our data show that for the heavily nicked SWNT electrons can be confined in such a zero-dimensional dot just like the experimental finding. The resonance structures depend on the specific configuration of the system. At different nicking strength, the nicked SWNT exhibit resonant and antiresonant behavior with large density of states (DOS). Near the resonance and antiresonance, the emittance $E_{12}$ is posi- tive giving rise to an inductivelike behavior. While at off resonances, the emittance is negative which corresponds to a capacitivelike response to the ac bias.

Our model Hamiltonian is a tight-binding model which is believed to provide a good description of the electronic structure of SWNT's. ${ }^{13}$ The method relies on a localizedorbital basis and it has been previously applied to study dynamic conductance and electronic transport properties of a number of nanotube systems. ${ }^{14}$ In this standard model, one $\pi$ electron orbital per carbon atom is kept, with zero on-site energies, whereas constant nearest-neighbor carbon bond hopping integral is chosen as $-3.0 \mathrm{eV}$. In the calculation, we assume that strong electron-electron interactions may be neglected.

To investigate ac conductance of the nicked SWNT at small frequencies, one can expand the dynamic conductance to the first order in frequency ${ }^{10}$

$$
G_{\alpha \beta}(\omega)=G_{\alpha \beta}(0)-i \omega q^{2} E_{\alpha \beta}+O\left(\omega^{2}\right),
$$

where the first term is the dc conductance $G_{12}=G$ and the second term is the emittance which contains the contributions from both the conduction current and the displacement current when the system is under ac bias. The presence of the displacement current is to ensure that the current is conserved. ${ }^{10}$ The emittance $E_{\alpha \beta}$ is defined as ${ }^{10}$

$$
\begin{aligned}
E_{\alpha \beta}= & \frac{d N_{\alpha \beta}}{d E}-D_{\alpha \beta}=\int d^{3} x \frac{d n_{\alpha \beta}(x)}{d E} \\
& -\int d^{3} x \frac{d n_{\beta}(x) / d E d \bar{n}_{\alpha}(x) / d E}{d n(x) / d E} .
\end{aligned}
$$

Note that $E_{\alpha \beta}$ satisfies the current conservation and gauge invariant condition $\Sigma_{\alpha} E_{\alpha \beta}=\Sigma_{\beta} E_{\alpha \beta}=0$. In Eq. (2) $d N_{\alpha \beta} / d E=\int d x\left[d n_{\alpha \beta}(x) / d E\right]$ is the global partial DOS (PDOS). Here the local PDOS $d n_{\alpha \beta} / d E$ is defined using the Green's function, ${ }^{15}$

$\frac{d n_{\alpha \beta}(x)}{d E}=\operatorname{Re}\left(\delta_{\alpha \beta}\left[G^{r} \Gamma_{\alpha} G^{r}\right]_{x x}+i\left[G^{r} \Gamma_{\beta} G^{a} \Gamma_{\alpha} G^{r}\right]_{x x}\right)$.

In terms of local PDOS the injectivity is defined as $d n_{\beta}(x) / d E=\Sigma_{\alpha} d n_{\alpha \beta}(x) / d E=\left[G^{r} \Gamma_{\beta} G^{a}\right]_{x x}$, and the emis- 

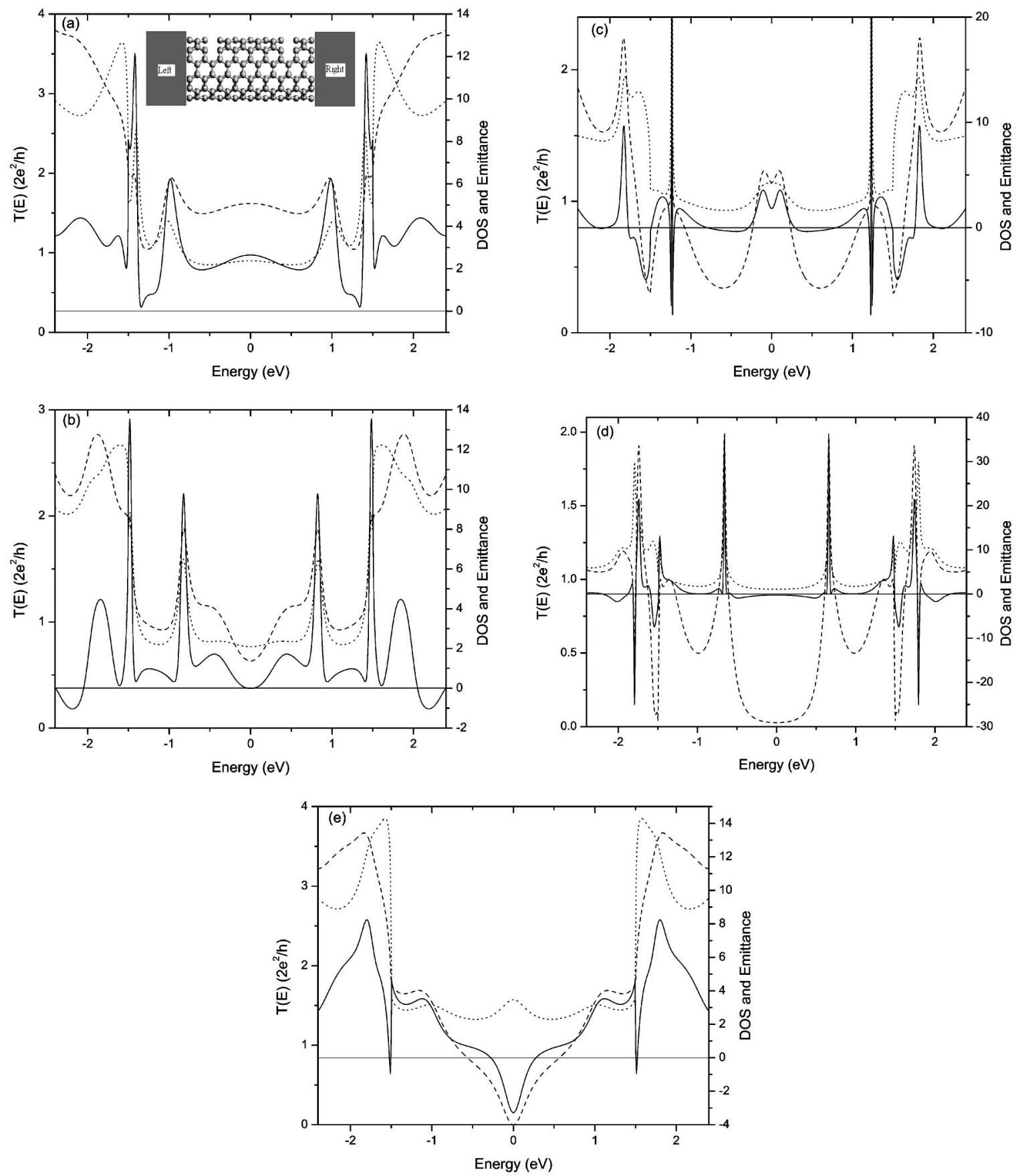

FIG. 1. The emittance (solid line), DOS (dotted line), and transmission coefficient (dashed line) vs Fermi energy for different nicked SWNT. Here (a)-(e) correspond to extracting two, four, six, eight, and three carbon atoms at each side of carbon rings. Inset of Fig. 1(a): a SWNT $(6,6)$ is nicked by extracting four carbon atoms at two sides.

sivity is $d \bar{n}_{\alpha}(x) / d E=\Sigma_{\beta} d n_{\alpha \beta}(x) / d E=\left[G^{a} \Gamma_{\alpha} G^{r}\right]_{x x}$. Finally, the total local DOS is $d n(x) / d E=\Sigma_{\alpha \beta} d n_{\alpha \beta}(x) / d E$ $=\left[G^{r} \Gamma G^{a}\right]_{x x}$, where the total linewidth function is defined as $\Gamma=\Gamma_{L}+\Gamma_{R}$ and $[A]_{x x}$ denotes the diagonal element of the relevant matrix. Two different behaviors can be observed depending on the sign of the emittance $E_{12} \cdot{ }^{10}$ For a single channel transmission, the system shows a capacitivelike behavior if $E_{12}$ is negative. Otherwise, the system is dominated by inductivelike behavior.

Now we proceed to calculate the transport properties of nicked SWNT. In order to find the relation between the spe- cific configuration of the nicked SWNT and their transport properties, we start by examining different nicked nanotubes. Taking into account a region consisting of 17 carbon rings (8.5 SWNT unit cells), we thus define the whole system as the central scattering region [inset of Fig. 1(a)] along with two semiinfinite leads. The calculations were carried out on $(6,6)$ nanotubes where about 200 carbon atoms in the central region, with both symmetric and asymmetric nicks on these metallic nanotubes. In Fig. 1, we plotted the evolution of the conducting behaviors of the nicked SWNT's under a variety of nicks along the circumference of the SWNT's. From Fig. 

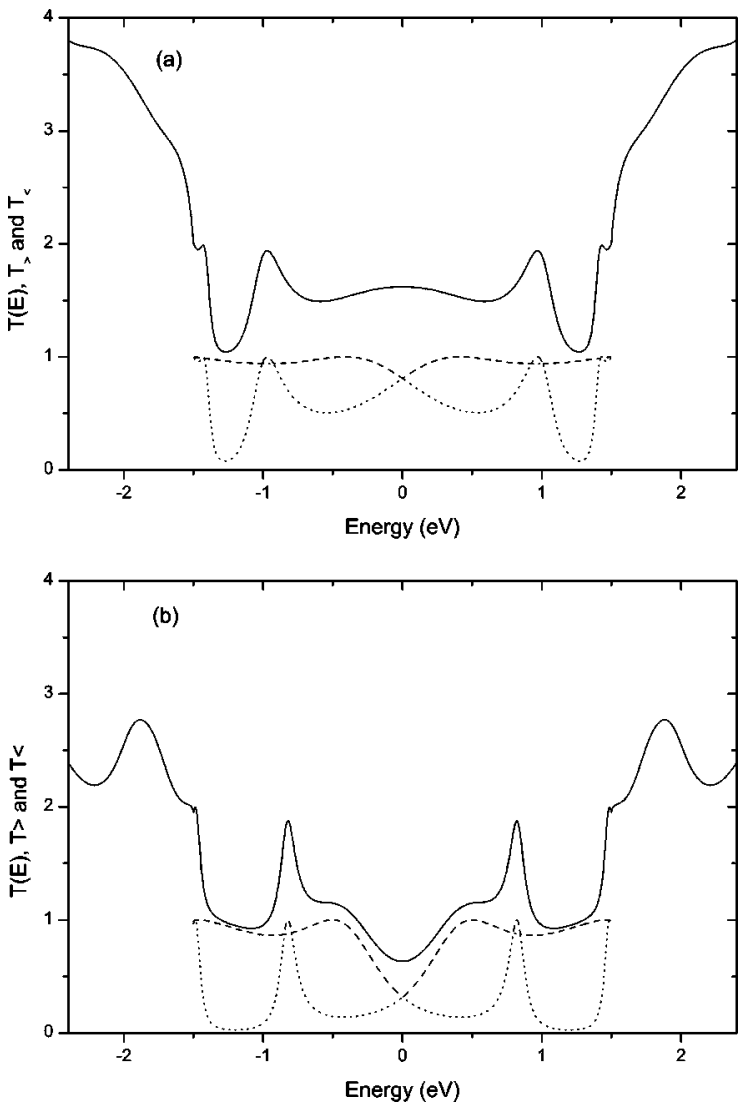

FIG. 2. Total transmission coefficient (solid line) and two transmission eigenchannels vs Fermi energy for different nicking configurations. Here (a) and (b) correspond to extracting two and four carbon atoms at each side of carbon rings, respectively.

1(a) to Fig. 1(d), the number of carbon atoms extracted from a carbon ring is $2,4,6,8 .{ }^{16}$ An apparent and understandable crossover from the ballistic to resonant transport has been demonstrated. For a perfect carbon nanotubes, there are two conducting channels near the Fermi level and the conductance is two within the first subband and becomes six beyond that. As the light nicks are introduced [Figs. 1(a) and 1(b)], the conductance decreases while maintaining high transmission background except near certain energies when the conductance remains about two. This can be understood as follows. When the SWNT is nicked, the constrictions are introduced. This in turn creates an effective barrier in SWNT since electrons cannot propagate in the nicked region (carbon ring). As a result, quasibound states may be established in the nicked SWNT which exhibit tunneling behavior shown in Figs. 1(a) and 1(b). To further understand the transmission behavior of nicked SWNT's, we focus on the first subband $|E|<1.5 \mathrm{eV}$. In this energy range there are only two eigenchannels and it is easy to obtain the separated contributions of these two channels by solving the combined equations ${ }^{17}$ $T=T_{>}+T_{<}$and $\operatorname{Tr}\left[t t^{\dagger} t t^{\dagger}\right]=T_{>}^{2}+T_{<}^{2}$. We see that in the case of light nicking (Fig. 2), one of the transmission channel is partially blocked and hence is in the resonant tunneling regime with two resonant peaks while the other channel is very transmissive with transmission coefficient close to one. Now we examine the emittance $E_{12}$ as a function of Fermi energy (solid lines in Fig. 1). We have also plotted the total global DOS for comparison (dotted line). In the case of light nicking [Figs. 1(a) and 1(b)], the system is fairly transmissive and hence the emittance is inductivelike. However, we see that at certain energies when one conductance channel is blocked the emittance decreases drastically and is close to zero. The line shape of emittance near the resonance of a single channel has been obtained analytically ${ }^{11}$ showing a positive resonant peak at the resonance and two negative ones near the resonance. The magnitudes of these positive and negative peaks depend on the coupling between the nicked SWNT and leads $\Gamma_{\alpha}$, which in turn depend on the sharpness of the resonant transmission peak. The line shape obtained here is similar to that of the single channel case except that the emittance is positive around the resonance. This is because the other nonresonant transmissive channel has positive contribution. We also observed a negative emittance for energy beyond the first subband, i.e., when $|E|$ $>1.5 \mathrm{eV}$. Similar to Fig. 1(b), for the energy $|E|$ between 1.5 and $2 \mathrm{eV}$, we also observed negative emittance. As two more atoms are removed on each carbon ring [Fig. 1(c)], similar behavior is observed with much sharper resonant peaks. Meanwhile the background transmission is suppressed significantly. In the case of medium nicking [Fig. 1(d)], a energy gap is opened up near the energy $E=0$ for both eigenchannels. Note that when the energy $|E|$ is between 1.5 and $2 \mathrm{eV}$, the transmission coefficient and the emittance are well correlated while the DOS behaves differently. For instance, in Fig. 1(d), near $|E|=1.8 \mathrm{eV}$, for the transmission dip, there is a peak in the DOS. This is the typical antiresonant behavior since a large DOS corresponds to a large dwell time ${ }^{19}$ which is the indicator of an anti-resonant state when transmission is small. ${ }^{20}$ Thus we tend to conclude that in Figs. 1(b) $-1(\mathrm{~d})$ at least one out of six conduction channels establishes an antiresonant state and the emittance becomes negative as a result. Finally, for the heavy nicking (removing 10 atoms), we found that one eigenchannel is completely blocked and the other one shows sharp resonant feature at resonant levels. Therefore, the calculated emittance agrees with the single-channel line shape. ${ }^{18}$ So far, we have removed even number of atoms. If odd number of atoms are removed (from 1 to 11 atoms), we found that the conductance is quenched at $E_{F}=0$, i.e., the transmission coefficient is zero at $E_{F}=0$. The corresponding emittance is negative which shows the capacitivelike behavior. For instance, in Fig. 1(e), we show the emittance, DOS, and transmission coefficient vs Fermi energy as three atoms are removed. In the medium and heavy nicking with 7-11 atoms removed, the resonant peaks start to emerge with emittance similar to case of removing even number of atoms.

Now we look at the asymmetric nicks which is more likely to represent the experimental result, since we may not be sure whether the nicks are always symmetric. If the nicks are asymmetric (see Fig. 3), the incoming electron will be totally blocked at some energies near the threshold of the first subband $|E|=1.3 \mathrm{eV}$. Our numerical results (not shown in the paper) indicate that one eigenchannel exhibits resonant behavior and the other channel is blocked significantly. Note that since the nicking is asymmetric the transmission coeffi- 


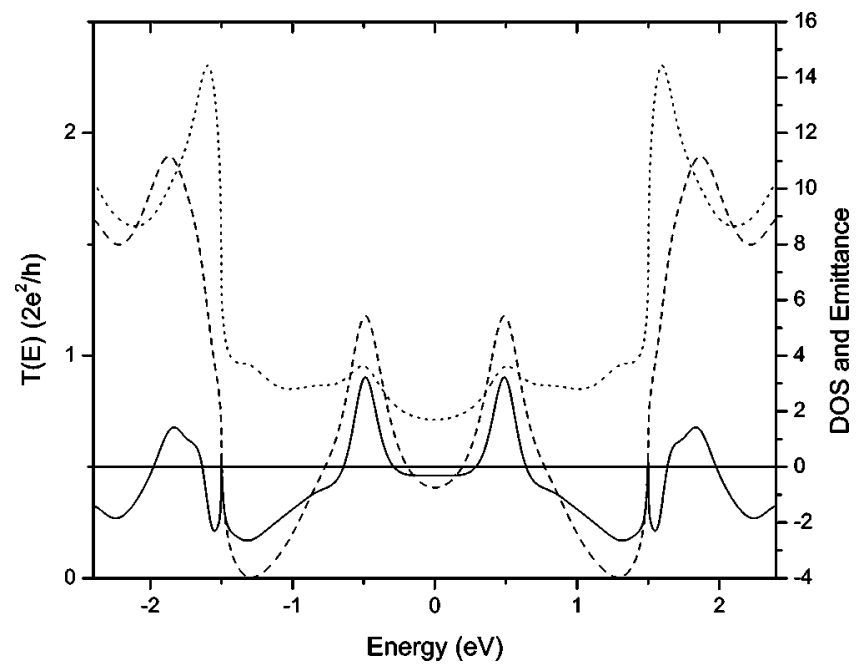

FIG. 3. The same as Fig. 1 but for an asymmetric nicked SWNT where four atoms are extracted from the left carbon ring and six atoms are nicked from the right.

cient of the resonant peak is less than one. In general, Figs. 1 and 3 provide a kind of selective transport filtering for the particular energy values in the case of medium and high nicking strength. The filter effect is not significant if the symmetric nicking strength is weak. When the system is asymmetric [configuration of Fig. 3 is a combination of configurations of Figs. 1(b) and 1(c)], the emittance of the behavior is different from that of Figs. 1(b) and 1(c). When the sym- metry is broken, the system responds capacitively in the larger energies. We see that the emittance near $E=0$ displays a flat region due to the suppression of the transmission coefficient. Near the edge of the first subband, the transmission coefficient raises abruptly with large DOS. As a result, the peak of emittance becomes positive.

In summary, we have calculated the transport properties through the quantum devices composed of nicked SWNT's. Several nicking strengths are considered and quantum dot behavior has been found only for heavily nicked carbon nanotubes. From our analysis, we found that near the resonances or antiresonances, the emittance $E_{12}$ is positive indicating that the system responds inductively. While at off resonances, the emittance $E_{12}$ is negative showing the capacitivelike response. Apart from this, when the nicks are slight the overall emittance $E_{12}$ is positive indicating that the relatively transparent conductor. As we increase the nicking strength, the capacitive characteristics appears for the smaller transmission probability. In the asymmetric case, same mechanism leads to a very weakly leaking capacitor except at the resonant energies. Furthermore, we have shown that the symmetry of the nicks on the SWNTs has a strong influence on the electronic transport properties and may not be neglected in the modeling of such a nanoscale device.

We gratefully acknowledge financial support from RGC grant (Grant No. HKU 7091/01P) from the government SAR of Hong Kong and a CRCG grant from the University of Hong Kong.
*Electronic address: jianwang@hkusub.hku.hk

${ }^{1}$ S. Iijima, Nature (London) 354, 56 (1991).

${ }^{2}$ Z. Yao et al., Nature (London) 402, 273 (1999); K. Tsukagoshi et al., ibid. 401, 572 (1999); S.J. Tans et al., ibid. 386, 474 (1997); S. Frank et al., Science 280, 1744 (1998); A.F. Morpurgo et al., ibid. 286, 263 (1999); D.H. Cobden et al., Phys. Rev. Lett. 81, 681 (1998); M.P. Anantram and T.R. Govindan, Phys. Rev. B 58, 4882 (1998); K. Kong et al., ibid. 60, 6074 (1999); H. Mehrez et al., Phys. Rev. Lett. 84, 2682 (2000); Phys. Rev. B 63, 245410 (2001); C. Roland et al., Phys. Rev. Lett. 84, 2921 (2000); Surf. Rev. Lett. 7, 637 (2000); A. Buldum and J.P. Lu, Phys. Rev. B 63, 161403 (2001); D. Orlikowski et al., ibid. 63, 155412 (2001); Y.D. Wei et al., ibid. 64, 115321 (2001); M. Brandbyge et al., ibid. 65, 165401 (2002).

${ }^{3}$ S.J. Tans et al., Nature (London) 393, 49 (1998).

${ }^{4}$ G. Cuniberti et al., Appl. Phys. Lett. 81, 850 (2002).

${ }^{5}$ R. Gutierrez et al., cond-mat/0211506, Chem. Phys. Chem. 4, 1252 (2003).

${ }^{6}$ S. Tans et al., Nature (London) 386, 474 (1997); M. Bockrath et al., Science 275, 1922 (1997).

${ }^{7}$ J.Y. Park et al., Appl. Phys. Lett. 80, 4446 (2002).

${ }^{8}$ For discussions on gauge invariance, see M. Büttiker and T. Christen, in Quantum Transport in Semiconductor Submicron Structures, edited by B. Kramer (Kluwer Academic, Dordrecht, 1996), pp. 263; Z.S. Ma et al., Phys. Rev. B 57, 9108 (1998); ibid. 59, 7575 (1999).

${ }^{9}$ M. Büttiker et al., Phys. Rev. Lett. 70, 4114 (1993).
${ }^{10}$ M. Buttiker, J. Phys.: Condens. Matter 5, 9361 (1993).

${ }^{11}$ M. Buttiker et al., Z. Phys. B: Condens. Matter 94, 133 (1994).

${ }^{12}$ T. Christen and M. Buttiker, Phys. Rev. B 53, 2064 (1996); J. Wang and H. Guo, ibid. 54, R11 090 (1996); Q.R. Zheng et al., ibid. 56, 12462 (1997); C.C. Wan, et al., ibid. 55, R13 393 (1997); W.D. Sheng et al., ibid. 59, 538 (1999); T. Gramespacher and M. Buttiker, ibid. 60, 2375 (1999); T. De Jesus et al., ibid. 62, 10774 (2000).

${ }^{13}$ X. Blase et al., Phys. Rev. Lett. 72, 1878 (1994); Y.A. Krotov et al., ibid. 78, 4245 (1997); L. Chico et al., ibid. 76, 971 (1996); V.H. Crespi et al., ibid. 79, 2093 (1997).

${ }^{14}$ M. Buongiorno Nardelli, Phys. Rev. B 60, 7828 (1999); H.J. Choi et al., Phys. Rev. Lett. 84, 2917 (2000); J. Taylor et al., Phys. Rev. B 63, 245407 (2001); Y.D. Wei et al., ibid. 63, 195412 (2001); Y.D. Wei and J. Wang, ibid. 66, 195419 (2002).

${ }^{15}$ T. Gramespacher and M. Buttiker, Phys. Rev. B 56, 13026 (1997).

${ }^{16}$ Removing several atoms leads to the appearance of dangling bonds and reconstruction of the damaged region. We assume that the tight-binding model can still be used approximately in this case.

${ }^{17}$ M. Branbyge et al., Phys. Rev. B 56, 14956 (1997); D.H. Kim et al., ibid. 64, 115409 (2001).

${ }^{18}$ M.K. Yip et al., Z. Phys. B: Condens. Matter 104, 463 (1997).

${ }^{19}$ M. Buttiker, Phys. Rev. B 27, 6178 (1983).

${ }^{20}$ J. Wang et al., Appl. Phys. Lett. 65, 1793 (1994). 